\title{
Association and Discriminatory Power of Abdominal Obesity for the Prevention of Sarcopenia and Vice Versa in Postmenopausal Women
}

\author{
Francisco José Gondim Pitanga ${ }^{1}$, Cristiano Penas Seara Pitanga ${ }^{2}$, \\ Ronaldo Eugénio Calçada Dias Gabriel ${ }^{3}$, Carmem Cristina Beck ${ }^{4}$, \\ Maria Helena Rodrigues Moreira ${ }^{5}$ \\ ${ }^{1}$ Department of Physical Education, Federal University of Bahia, Salvador, Brazil \\ ${ }^{2}$ Integrated Institute of Health (SPORTCLIN), Salvador, Brazil \\ ${ }^{3}$ Centre for the Research and Technology of Agro-Environmental and Biological Sciences (CITAB), Laboratory of Biomechanics, Body \\ Composition and Health (LaB2Health), University of Trás-os-Montes and Alto Douro, Vila Real, Portugal \\ ${ }^{4}$ Federal Institute of Santa Catarina, Palhoça, Brazil \\ ${ }^{5}$ Research Center in Sports Sciences, Health Sciences and Human Development (CIDESD), Centre for the Research and Technology of \\ Agro-Environmental and Biological Sciences (CITAB), Laboratory of Biomechanics, Body Composition and Health (LaB2Health), \\ University of Trás-os-Montes and Alto Douro, Vila Real, Portugal
}

Email address:

pitanga@lognet.com.br (F. J. G. Pitanga)

\section{To cite this article:}

Francisco José Gondim Pitanga, Cristiano Penas Seara Pitanga, Ronaldo Eugénio Calçada Dias Gabriel, Carmem Cristina Beck, Maria Helena Rodrigues Moreira. Association and Discriminatory Power of Abdominal Obesity for the Prevention of Sarcopenia and Vice Versa in Postmenopausal Women. European Journal of Preventive Medicine. Vol. 6, No. 5, 2018, pp. 70-75. doi: 10.11648/j.ejpm.20180605.12

Received: October 6, 2018; Accepted: October 19, 2018; Published: November 10, 2018

\begin{abstract}
Both abdominal obesity and sarcopenia are associated with cardiometabolic disorders mainly in postmenopausal women. In addition, there appears to be a reverse association between abdominal obesity and sarcopenia. The objective of the study was to identify the association and the discriminatory power of abdominal obesity for the prevention of sarcopenia and vice versa in postmenopausal women. The sample includes 257 women with age 57,2 years, The abdominal obesity and the variables determinants of sarcopenia were assessed by bioelectrical impedance analysis. The association of variables was been tested using logistic regression and the discriminatory power through Receiver Operating Characteristic (ROC) considering a confidence interval of $95 \%$. The presence of abdominal obesity with the cutoff point of $153 \mathrm{~cm}^{2}$ of visceral fat was discriminatory power for sarcopenia, as well as the musculoskeletal mass index with the cuttof point of $35,2 \%$ discriminated the absence of abdominal obesity. After adjusting for confounders it was observed still association between abdominal obesity and sarcopenia and association between musculoskeletal mass index and absence of abdominal obesity. The results of the study suggest that abdominal obesity discriminates and is associated with sarcopenia, such as musculoskeletal mass index discriminates the absence and is inversely associated whit of abdominal obesity in postmenopausal women.
\end{abstract}

Keywords: Abdominal Obesity, Sarcopenia, Postmenopausal Women

\section{Introduction}

Abdominal obesity is a strong risk factor for cardiometabolic disorders, and may also be associated with sarcopenia, that is, loss of muscle protein associated with a decline in muscle strength and functional quality of the muscle. The inverse association is also possible, considering that a decrease in musculoskeletal mass may cause abdominal obesity [1].

The mechanisms by which abdominal obesity increases metabolic and cardiovascular risk can be partially explained by excessive free fatty acid production in the visceral abdominal region, which is distributed by portal circulation. This exposes the liver to high fat concentrations and reduces 
hepatic insulin removal, resulting in peripheral hyperinsulinemia and a consequent rise in the risk of developing diabetes mellitus. Hyperinsulinemia can also cause disturbances in plasma lipid concentrations, as well as hypertension [2].

On the other hand, increased abdominal adiposity induces chronic inflammation, which may contribute to the development and progression of sarcopenia. Deregulation of adipocytokines produced in visceral adipose tissue, such as the alpha tumor necrosis factor (TNF- $\alpha$ ), interleukin-6 (IL-6), leptin and adiponectin, has been reported to influence insulin resistance and growth hormone secretion, which is closely related to sarcopenia. Insulin is an anabolic factor and insulin resistance is considered the main factor explaining the relationship between obesity and sarcopenia [1, 3].

Sarcopenia can also cause abdominal obesity, considering that a decline in skeletal muscle mass decreases energy expenditure, causing greater body fat concentration, primarily in the abdominal region [4]. Moreover, individuals with less muscle mass are at increased risk of hepatic steatosis [5], hypertension and hardened arteries [6].

This may have important consequences in postmenopause, since women undergo significant changes related to their social role during this phase. It also causes uncomfortable symptoms and an increase in disease prevalence, including cardiovascular disease, hypertension, lipid alterations and diabetes, which can stem from excessive visceral fat and sarcopenia [7].

As such, the aim of this study was to identify the association and discriminatory power of abdominal obesity for sarcopenia and vice versa in postmenopausal women.

\section{Methods}

\subsection{Study Design}

The study is part of the project entitled "Menopause in Shape", approved by the Science and Technology Foundation (POCI/DES/59049/2004) and the University of Trás-osMontes and Alto Douro (UTAD, Portugal), aimed at analyzing the effects of physical exercise on cardiovascular risk, falling and the physical and functional aptitude of postmenopausal women [8].

The participants volunteered to take part in the project in response to different publicity campaigns (newspaper ads, pamphlets, television reports, among others) or after being referred by their family doctor. The sample was recruited between November 2005 and March 2006 and inclusion was preceded by assessment of their clinical and reproductive history. The study complied with Declaration of Helsinki procedures [9], and all the subjects gave their informed consent. The protocol was not submitted to the UTAD Ethics Committee because it was only established in 2012 .

Assessments were conducted at the UTAD Laboratory of Physical Aptitude, Exercise and Health by two trained technicians supervised by the principal investigator of the project.

\subsection{Sample}

The sample consisted of 257 postmenopausal women with average age of $57.2 \pm 6.6$ years. The following inclusion criteria were adopted: (a) absence of early menopause; (b) no significant kidney, liver or hematological disease; (c) no symptoms of angina or myocardial infarction in the previous 3 months; (d) absence of uncontrolled hypertension (systolic blood pressure $\geq 200 \mathrm{mmHg}$ and/or diastolic blood pressure $\geq 105 \mathrm{mmHg}$ ); (e) not using beta blockers or antiarrhythmic drugs and (f) no musculoskeletal conditions precluding exercise or exacerbated by it.

Menopause was classified as natural when menstrual and ovulatory cycles occurred without clear pathological cause and permanent amenorrhea existed for at least 12 months. Time with menopause $(1, \mathrm{TM} \leq 10$ years; $2, \mathrm{TM}>10$ years $)$, the use of hormone therapy (HT) $(0$, no documented use of HT; 1 documented use of HT), the use of contraceptive methods (0, did not use; 1 , used) and regular menstrual cycles in the reproductive stage $(0$, no; 1 , yes $)$ were expressed on an ordinal scale. The last variable was defined by the presence of consistent menstrual cycles (between 21 and 35 days).

\subsection{Instruments and Procedures}

Height (HT) was measured with a SECA 220 stadiometer (Seca Corporation, Hamburg Germany), in accordance with the procedures established by Heyward and Wagner (2004) [10] and a tolerance limit of $2 \mathrm{~mm}$ was considered. Weight (W), visceral fat area (VFA) and skeletal muscle mass (SMM) were assessed using an InBody720 body composition analyzer (Biospace Co, Ltd, Seoul, Korea), in line with the procedures contained in the device's manual [11] and guidelines published in the literature [12]. This equipment uses eight electrodes ( 4 on the palms and thumbs and 4 on the heels and the front of the feet) in order to analyze impedance in 5 regions of the body (upper limbs, torso and lower limbs), measuring resistance at 6 frequencies $(1 \mathrm{kHz}, 5$ $\mathrm{kHz}, 50 \mathrm{kHz}, 250 \mathrm{kHz}, 500 \mathrm{kHz}$, and $1000 \mathrm{kHz}$ ) and reactance at 3 frequencies $(5 \mathrm{kHz}, 50 \mathrm{kHz}$, and $250 \mathrm{kHz}$ ). Assessments were made by the same technician, and body contact points with the electrodes were cleaned with an electrolytic cloth recommended by the manufacturer. The data were imported to Excel, using Lookin'Body 3.0 software (Biospace Co, Ltd, Seoul, Korea). The skeletal muscle mass index (SMMI) was calculated according to the formula $\mathrm{SMMI}=\mathrm{SMM} \times 100$, proposed by Janssen, Heymsfield and Ross, 2002 [13], considering whether sarcopenia exists in the presence of SMMI $\leq 28 \%$. The validity of the InBody 720 in estimating total and segmental body composition is documented in the literature [14]. The technical errors of W, HT and SMM obtained in duplicate measures of 10 postmenopausal women and via application of the formula $\mathrm{TE}=\left(\sum \mathrm{d}^{2} / 2 \mathrm{n}\right)^{0.5}(\mathrm{~d}$, difference between the two assessments; n, number of sample elements), were $0.06 \mathrm{~kg}$, $0.09 \mathrm{~cm}$ and $0.20 \mathrm{~kg}$, respectively. 


\subsection{Statistical Analysis}

The data were analyzed using the SATA statistical program (version 7.0, Stata Corp, College Station, USA). The discriminatory power and cutoff points for physical activity to prevent sarcopenia were analyzed using Receiver Operating Characteristic (ROC) curves.

First, it was analyzed the total area under the ROC curve between abdominal obesity and prevention of sarcopenia and vice versa, observing a larger area associated with discriminatory power. In this stage, a $95 \%$ confidence interval (CI) was used, with a lower bound not less than 0.50 . Next, sensitivity and specificity were calculated, in addition to the cutoff points for abdominal obesity to prevent sarcopenia and vice versa. Stratification was conducted to determine the presence of effect modifiers and confounders, observing stratum-specific measures and their confidence intervals. Considering a $95 \%$ confidence interval using the Mantel-Haenzel method, analysis for confounders was conducted comparing the odds ratio (OR) between the crude association and that adjusted for possible confounders, considering $10 \%$ as the identification parameter of the difference between the aforementioned associations.

Logistic regression analysis was carried out applying the backward method, starting with the complete model and removing possible confounding variables one by one, which, when eliminated from the model, could cause a $10 \%$ or greater change in the association between abdominal obesity and sarcopenia and vice versa [15]. Finally, OR was estimated using the model that best explained the association. The following covariables were analyzed for possible modifying or confounding effects: age, time with menopause, hormone therapy, nature of the menopause $(0$, natural; 1 , induced), menstrual cycle regularity and the use of contraceptive methods.

\section{Results}

Sample characteristics are illustrated in Table 1.

Table 1. Descriptive analysis of the sample $(n=257)$.

\begin{tabular}{lll}
\hline Variables & Mean/Median* \pm SD or percentage & Amplitude or confidence interval \\
\hline Age (years) & $57.2 \pm 6.6$ & $40.6-79.6$ \\
Weight (kg) & $69.1 \pm 11.3$ & $45.8-108.7$ \\
Height (cm) & $155.0 \pm 5.2$ & $142-170$ \\
Skeletal muscle mass (kg) & $22.3 \pm 2.8$ & $15.8-30.4$ \\
Skeletal muscle mass index (\%) & & \\
$\geq 28$ (normal muscle condition) & 90.7 & $(86.4-93.9)$ \\
$<28$ (sarcopenia) & 9.3 & $(6.1-13.6)$ \\
Visceral fat area $\left(\mathrm{cm}^{2}\right)$ & & \\
$<100 \mathrm{~cm}^{2}(\%)$ & 6.193 .9 & $(3.3-9.7)$ \\
$\geq 100 \mathrm{~cm}^{2}(\%)$ & & $(90.2-96.6)$ \\
Menstrual period regularity (\%) & 15.0 & $(10.9-20.3)$ \\
No & 85.0 & $(80.2-89.4)$ \\
Yes & 28.4 & $(22.9-34.4)$ \\
Use of contraceptive methods (\%) & 71.6 & $(65.6-77.1)$ \\
No & & $(39.7-52.4)$ \\
Yes & 45.9 & $(47.6-60.3)$ \\
Hormone therapy (\%) & 54.1 & $(52.8-65.3)$ \\
No & & $(34.6-47.2)$ \\
Yes & 59.2 & $(69.3-80.4)$ \\
Time with menopause (\%) & 40.8 & $(19.6-30.6)$ \\
$\leq 10$ years & 75.2 & \\
$>10$ years & 24.8 & \\
Nature of the menopause (\%) & & \\
Natural & & \\
Induced & &
\end{tabular}

SD-standard deviation

Table 2 and Figures 1 and 2 show the areas under the ROC curves between abdominal obesity and sarcopenia and between the skeletal muscle mass index and absence of abdominal obesity. Statistically significant areas were observed for both abdominal obesity as discriminator of sarcopenia, and the skeletal muscle mass index as discriminator of the absence of abdominal obesity.

Table 2. Area under the ROC curve between abdominal obesity and sarcopenia and between skeletal muscle mass index and absence of abdominal obesity.

\begin{tabular}{lll}
\hline Body composition variables & Area & CI (95\%) \\
\hline Abdominal obesity and sarcopenia & 0.90 & $0.85-0.96$ \\
Skeletal muscle mass index and absence of abdominal obesity & 0.96 & $0.93-0.98$ \\
\hline
\end{tabular}

CI: confidence interval 


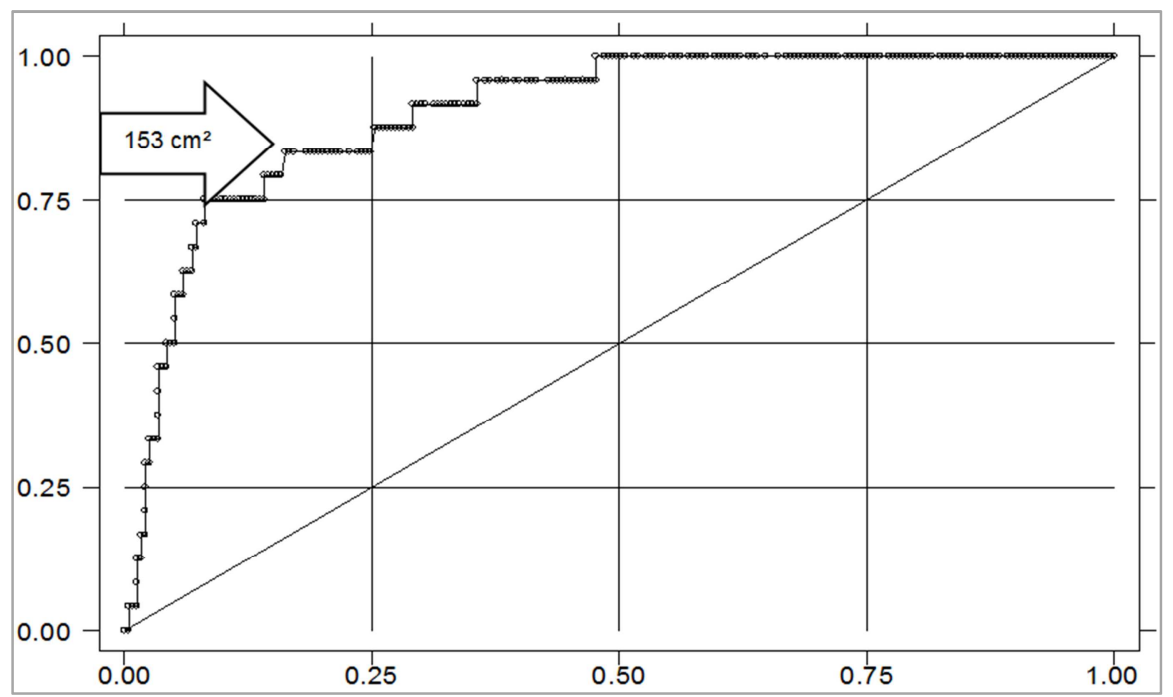

Figure 1. Area under the ROC curve, with its respective cutoff point, between abdominal obesity and sarcopenia in postmenopausal women.

Table 3 and Figures 1 and 2 contain the cutoff points and their respective sensitivities and specificities of abdominal obesity as discriminator of sarcopenia and the skeletal muscle mass index as discriminator of the absence of abdominal obesity.
Table 3. Cutoff points, sensitivity and specificity of abdominal obesity as discriminator of sarcopenia and skeletal muscle mass index as discriminator of absence of abdominal obesity.

\begin{tabular}{llll}
\hline Variables & Cutoff point & Sensitivity & Specificity \\
\hline Abdominal obesity & $153 \mathrm{~cm}^{2}$ & $83 \%$ & $83 \%$ \\
Skeletal muscle mass index & $35.2 \%$ & $100 \%$ & $85.4 \%$ \\
\hline
\end{tabular}

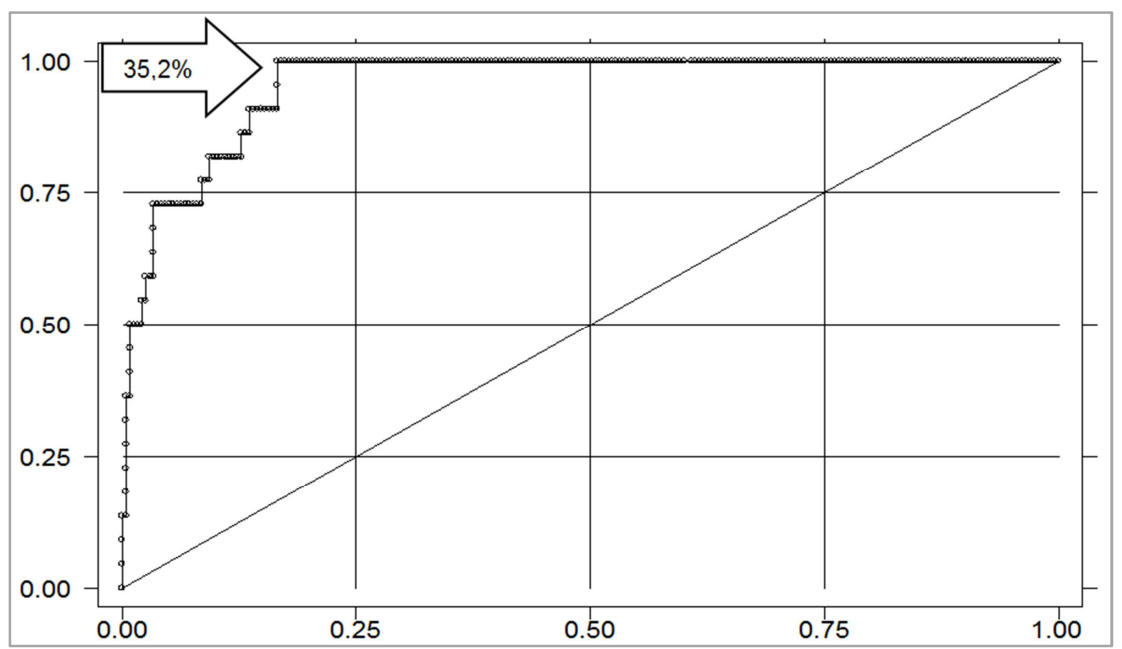

Figure 2. Area under the ROC curve, with its respective cutoff point, between skeletal muscle mass index and abdominal obesity in postmenopausal women.

Table 4 exhibits the associations between abdominal obesity and sarcopenia and between the skeletal muscle mass index and abdominal obesity. There is a strong association between abdominal obesity and sarcopenia and an inverse association between the skeletal muscle mass index and abdominal obesity.

Table 4. Association between abdominal obesity and sarcopenia and between skeletal muscle mass and abdominal obesity.

\begin{tabular}{lcl}
\hline Variables & OR & $\mathbf{( 9 5 \% ~ C I ) ~}$ \\
\hline Abdominal obesity & 35.0 & $8.85-138.6$ \\
Skeletal muscle mass index & 0.48 & $0.38-0.60$ \\
\hline
\end{tabular}

*Association adjusted for time with menopause, menstrual cycle regularity, use of oral contraceptives, age, and hormone replacement.

\section{Discussion}

The study demonstrated the discriminatory power of abdominal obesity for sarcopenia and vice versa. It also showed the association between abdominal obesity and sarcopenia, in addition to the inverse association between the skeletal muscle mass index and abdominal obesity in postmenopausal women.

Thus, there is discriminatory power and an association in both directions, as suggested by Roubenoff in 2004 [1], who reported that a decline in muscle function reduces insulinsensitive tissue mass, resulting in insulin resistance, which promoted metabolic syndrome and obesity. The increase in 
adipose tissue, namely visceral, raises the production of adipokines such as IL-6 (decreases glucose transporters), TNF- $\alpha$ (reduces glucose and insulin transport) and resistance (limits the use of glucose in muscles) and causes a decline in visfatin synthesis (poor binding and activation of insulin receptors), generating tissue resistance to insulin with a direct catabolic effect on muscles. Thus, a vicious circle is created, leading to greater gains in adiposity and negatively affecting muscle condition.

Partially corroborating these results, a longitudinal study conducted with 379 Koreans of both sexes to investigate whether visceral fat influenced skeletal muscle mass and vice versa revealed that only visceral fat was associated with a decline in skeletal muscle mass. The association between skeletal muscle mass and visceral fat was not statistically significant [4]. The authors reported that, although skeletal muscle mass does not predict changes in visceral fat during the follow-up period, a longer period or larger number of participants could reveal skeletal muscle mass as an independent risk factor for the development of visceral obesity.

These results suggest the cutoff points for using visceral obesity as discriminator of sarcopenia $\left(153 \mathrm{~cm}^{2}\right)$ and the skeletal muscle mass index as discriminator of the absence of visceral obesity in postmenopausal women (35.2\%). These cutoff points could be used in diagnosing both abdominal obesity and sarcopenia and, taken together, serve as a screening tool for sarcopenic obesity.

Sarcopenic obesity was initially characterized as the copresence of sarcopenia and obesity, triggered by the complex interaction of physiopathological mechanisms, such as an increase in proinflammatory cytokines, oxidative stress, insulin resistance, hormonal changes and decreased physical activity [16]. It is important to underscore that we analyzed visceral but not total obesity.

In this respect, earlier studies found that visceral fat and thigh muscles can be used as an indicator of sarcopenia obesity and associated with metabolic syndrome [17]. The increase in fat mass may exacerbate sarcopenia because chronic lipid accumulation has a harmful effect on amino acid incorporation and muscle protein synthesis [18].

A possible study limitation is the instrument used to assess visceral fat by bioimpedance, which, despite having been applied in other work conducted by our research group [19, 20, 21], was not validated against a gold standard for assessing abdominal obesity.

\section{Conclusion}

The results of the study suggest that abdominal obesity discriminates and is associated with sarcopenia, and skeletal muscle mass index discriminates the absence and is inversely associated with abdominal obesity in postmenopausal women.

\section{Acknowledgements}

This study was financed by the European Investment
Funds via FEDER/COMPETE/POCI - Program of Competitiveness and Internationalization, as part of the project POCI-01-0145-FEDER-006958, and National Funds through the FCT - Science and Technology Foundation as part of the project UID/AGR/04033/2013

\section{References}

[1] Roubenoff R, Sarcopenic obesity: the confluence of two epidemics, Obes Res, 2004 Jun; 12(6): 887-8.

[2] Björntorp P, Adipose tissue distribuition and function, Int $\mathrm{J}$ Obes 1991; 15 Supp1 2: 67-81.

[3] Sakuma K, Yamaguchi A (2010) Molecular mechanisms in aging and current strategies to counteract sarcopenia, Curr Aging Sci 3: 90-101.

[4] Kim TN, Park MS, Ryu JY, Choi HY, Hong HC, et al, (2014) Impact of Visceral Fat on Skeletal Muscle Mass and Vice Versa in a Prospective Cohort Study: The Korean Sarcopenic Obesity Study (KSOS), PLoS ONE 9(12): e115407, doi: 10,1371/journal,pone, 0115407 .

[5] Hong HC, Hwang SY, Choi HY, et al, Relationship between sarcopenia and nonalcoholic fatty liver disease: the Korean Sarcopenic Obesity Study, Hepatology 2014; 59: 1772-1778.

[6] Han K, Park YM, Kwon HS, et al, Sarcopenia as a determinant of blood pressure in older Koreans: findings from the Korea National Health and Nutrition Examination Surveys (KNHANES) 2008-2010, PLoS One 2014; 9: e86902.

[7] Kimmerle R, Heinemann L, Heise T, Bender R, Weyer C, Hirschberger S, Berger M. Influence of continuous combined estradiol-norethisterone acetate preparations on insulin sensitivity in postmenopausal nondiabetic women. Menopause. 1999; 6(1): 36-42.

[8] Moreira, H, Influência do Exercício Físico no Risco Cardiovascular e na Aptidão Física e Funcional de Mulheres Pós-Menopáusicas, F, p, a, C, e, Tecnologia, Editor 2004: Vila Real.

[9] World Medical Association, World medical association declaration of Helsinki: ethical principles for medical research involving human subjects, ed, Seoul: WMA General Assembly, 2008.

[10] Heyward, V, H, and D, Wagner, R, Applied Body Composition Assessment, Second Edition ed, 2004, Champaign: Human Kinetics.

[11] Biospace, ed, InBody 720 the precision body composition analyser: user's manual, ed, L, Biospace Co, 2008, Biospace Co, Ltd,: Seoul.

[12] Chumlea, W, \& Sun, S, (2005), Bioelectrical impedance analysis, In S, Heymsfield, T, Lohman, Z, Wang \& S, Going (Eds,), Human body composition (pp, 79-88), Champaign: Human Kinetics.

[13] Janssen, I, Heymsfield, S, \& Ross, R, (2002), Low relative skeletal muscle mass (sarcopenia) in older persons is associated with functional impairment and physical disability, Journal of the American Geriatrics Society, 50(5), 889-896, doi: 10,1046/j,1532-5415,2002,50216,x. 
[14] Ling, C, Craen, A, Slagboom, P, Gunn, D, Stokkel, M, Westendorp, R, Maier, A, (2011), Accuracy of direct segmental multi-frequency bioimpedance analysis in the assessment of total body and segmental body composition in middle-aged adult population, Clinical Nutrition, 30(5), 610615, doi: 10,1016/j,clnu,2011,04,00.

[15] Hosmer, D, \& Lemeshow, S, (2005), Applied logistic regression $\left(2^{\mathrm{a}} \mathrm{ed},\right)$, New York: John Wiley \& Sons, Inc.

[16] Choi KM, Sarcopenia and sarcopenic obesity, Korean J Intern Med, 2016 Nov; 31(6): 1054-1060.

[17] Lim KI, Yang SJ, Kim TN, Yoo HJ, Kang HJ, et al, (2010) The association between the ratio of visceral fat to thigh muscle area and metabolic syndrome: the Korean Sarcopenic Obesity Study (KSOS), Clin Endocrinol (Oxf) 73: 588-594.
[18] Masgrau A, Mishellany-Dutour A, Murakami H, Beaufrere AM, Walrand S, et al, (2012) Time-course changes of muscle protein synthesis associated with obesity-induced lipotoxicity, J Physiol 590: 5199-5210.

[19] Pitanga CP, Pitanga FJ, Beck CC, Gabriel RE, Moreira MH, [Level of physical activity in the prevention of excess visceral fat in postmenopausal women: how much is needed?], Arq Bras Endocrinol Metabol, 2012; 56(6): 358-63.

[20] Pitanga FJ, Pitanga CP, Beck CC, Gabriel RE, Moreira MH, Anthropometry to identify high visceral fat area in postmenopausal women, Nutr Hosp, 2015; 32(6): 2535-9.

[21] Pitanga, C. P. S., Pitanga, F. J. G., Moreira, M. H. R., Gabriel, R. E. C. Associação entre o nível de atividade física e a área de gordura visceral emmulheres pós-menopáusicas. Rev. Bras. Med. Esporte. 2014; 20(4): 252-256. 